\title{
Self-affirmation model for football goal distributions
}

\author{
Elmar Bittner $^{1}$, Andreas Nußbaumer ${ }^{1}$, Wolfhard Janke $^{1}$ and Martin Weigel ${ }^{2}$ \\ ' ${ }^{1}$ Institut für Theoretische Physik and Centre for Theoretical Sciences (NTZ) - Universität Leipzig \\ Postfach 100 929, D-04009 Leipzig, Germany \\ 2 Department of Mathematics and the Maxwell Institute for Mathematical Sciences, Heriot-Watt University \\ Riccarton, Edinburgh, EH144AS, Scotland, UK
}

PACS 89.20.-a - Interdisciplinary applications of physics

PACS 02.50. $-r$ - Probability theory, stochastic processes, and statistics

\begin{abstract}
Analyzing football score data with statistical techniques, we investigate how the highly co-operative nature of the game is reflected in averaged properties such as the distributions of scored goals for the home and away teams. It turns out that in particular the tails of the distributions are not well described by independent Bernoulli trials, but rather well modeled by negative binomial or generalized extreme value distributions. To understand this behavior from first principles, we suggest to modify the Bernoulli random process to include a simple component of self-affirmation which seems to describe the data surprisingly well and allows to interpret the observed deviation from Gaussian statistics. The phenomenological distributions used before can be understood as special cases within this framework. We analyzed historical football score data from many leagues in Europe as well as from international tournaments and found the proposed models to be applicable rather universally. In particular, here we compare men's and women's leagues and the separate German leagues during the cold war times and find some remarkable differences.
\end{abstract}

Football (soccer) is of the most popular sports worldwide, attracting millions of spectators each year. Its popularity and economical importance also captivated scientists from many fields, for instance in the attempt to improve the game tactics etc. Much less effort has been devoted, it seems, to the understanding of football (and other ball sports) from the perspective of the stochastic behavior of co-operative "agents" (i.e., players) in abstract models. ' Such problems recently have come into the focus of physicists in the hope that the model-based point-of-view and methodological machinery of statistical mechanics might add a new perspective to the much more detailed investigations of more specific disciplines [1,2]. Some reports of such research are collected in Ref. [3]. Score distributions of ball games have been occasionally considered by statisticians [4-7]. Very small data sets were initially found to be reasonably well described by the simplest Poissonian model resulting from constant and independent scoring probabilities [4]. Including more data, however, better phenomenological fits were achieved with models such as the negative binomial distribution (NBD), which can be constructed from a mixture of independent Poissonian processes [6], or even with models of generalized extreme value (GEV) statistics $[7,8]$, which are particularly suited for heavy-tailed distributions. This yielded a rather inhomogeneous and purely phenomenological picture, without offering any microscopical justification. We argue that the crucial ingredient missed in previous studies are the correlations between subsequent scoring events.

In a broader context, this problem of extremes is of obvious importance, for instance, in actuarial mathematics and engineering, but the corresponding distributions with fat tails also occur in many physics fields, ranging from the statistical mechanics of regular and disordered systems [9-12] over turbulence [13] to earth quake data [14]. In these cases often average properties were considered instead of explicit extremes, and the empirical occurrence of heavy-tailed distributions led to speculations about hidden extremal processes, most of which could not be identified, though. It was only realized recently that GEV distributions can also arise naturally as the statistics of sums of correlated random variables [15], which could explain their ubiquity in nature.

For the specific example of scoring in football, correlations naturally occur through processes of feedback of scoring on both teams, and we shall see how the intro- 
duction of simple rules for the adaptation of the success probabilities in a modified Bernoulli process upon scoring a goal leads to systematic deviations from Gaussian statistics. We find simple models with a single parameter of self-affirmation to best describe the available data, including cases with relatively poor fits of the NBD. The latter is shown to result from one of these models in a particular limit, explaining the relatively good fits observed before.

To investigate the importance of correlations, we consider the distributions of goals scored by the home and away teams in football league or cup matches. To the simplest possible approximation, both teams have independent and constant (small) probabilities of scoring during each appropriate time interval of the match, such that the resulting final scores $n$ follow a Poisson distribution,

$$
P_{\lambda}(n)=\frac{\lambda^{n}}{n !} e^{-\lambda}
$$

where $\lambda=\langle n\rangle$. Here and in the following, separate parameters are chosen for the scores of the home and away teams. Clearly, this is a gross over-simplification of the situation. Averaging over the matches during one or several seasons, one might expect a distribution of scoring probabilities $\lambda$ depending on the different skills of the teams, the lineup for the match etc., leading to the notion of a compound Poisson distribution. For the special case of $\lambda$ following a gamma distribution $f(\lambda)$, the resulting compound distribution is a NBD [16],

$$
P_{r, p}(n)=\int_{0}^{\infty} \mathrm{d} \lambda P_{\lambda}(n) f(\lambda)=\frac{\Gamma(r+n)}{n ! \Gamma(r)} p^{n}(1-p)^{r} .
$$

The NBD form has been found to describe football score data rather well $[6,7]$. It appears rather ad hoc, however, to assume that $f(\lambda)$ follows a gamma form, and fitting different seasons of our data with the Poissonian model (11), the resulting distribution of $\lambda$ does not resemble a gamma distribution. As a phenomenological alternative to the NBD, Greenhough et al. [7] considered fits of the GEV distributions

$$
P_{\xi, \mu, \sigma}(n)=\frac{1}{\sigma}\left(1+\xi \frac{n-\mu}{\sigma}\right)^{-1-1 / \xi} e^{-\left(1+\xi \frac{n-\mu}{\sigma}\right)^{-1 / \xi}}
$$

to the data, obtaining clearly better fits than with the NBD in some cases. Depending on the value of the parameter $\xi$, these distributions are known as Weibull $(\xi<0)$, Gumbel $(\xi \rightarrow 0)$ and Fréchet $(\xi>0)$ distributions, respectively [8].

In the present context of scoring in football, goals are likely not independent events but, instead, scoring certainly has a profound feedback on the motivation and possibility of subsequent scoring of both teams (via direct motivation/demotivation of the players, but also, e.g., by a strengthening of defensive play in case of a lead). Such feedback can be taken into account starting from a simple Bernoulli model: consider a match divided into, e.g.,
$N=90$ time steps with both teams having the possibility to score in each unit with a probability $p=p(n)$ depending on the number $n$ of goals scored so far. Several possibilities arise. For our model "A", upon each goal the scoring probability is modified as $p(n)=p(n-1)+\kappa$, with some fixed constant $\kappa$. Alternatively, one might consider a multiplicative modification rule, $p(n)=\kappa p(n-1)$, which we refer to as model "B". Finally, in our model "C" the assumption of independence of the scoring of the two teams is relaxed by coupling the adaptation rules, namely by setting $p_{\mathrm{h}}(n)=p_{\mathrm{h}}(n-1) \kappa_{\mathrm{h}}, p_{\mathrm{a}}(n)=p_{\mathrm{a}}(n-1) / \kappa_{\mathrm{a}}$ upon a goal of the home (h) team, and vice versa for an away (a) goal. If both teams have $\kappa>1$, this results in an incentive for the scoring team and a demotivation for the opponent, but a value $\kappa<1$ is conceivable as well. The resulting, distinctly non-Gaussian distributions $P_{N}(n)$ for the total number of goals scored by one team can be computed exactly for models "A" and "B" from a Pascal recurrence relation [17],

$$
P_{N}(n)=[1-p(n)] P_{N-1}(n)+p(n-1) P_{N-1}(n-1),
$$

where $p(n)=p_{0}+\kappa n$ (model "A") or $p(n)=p_{0} \kappa^{n}$ (model "B"). Model "C" can be treated similarly [17].

It is remarkable that this rather simple class of feedback models leads to a microscopic interpretation of the NBD in (2) which, in fact, can be shown to be the continuum limit of $P_{N}(n)$ for model "A", i.e., $N \rightarrow \infty$ with $p_{0} N$ and $\kappa N$ kept fixed [17]. For the NBD parameters one finds that $r=p_{0} / \kappa$ and $p=1-\mathrm{e}^{-\kappa N}$, such that a good fit of a NBD to the data can be understood from the effect of self-affirmation of the teams or players, the major ingredient of our microscopic models "A", "B", and "C". Additionally, a certain type of continuous microscopic model with feedback can be shown to result in a GEV distribution $[15,17]$, such that all different types of deviations from the Gaussian form occurring here can be understood from the correlations introduced by feedback.

We now confront these models with empirical data sets, starting with football matches played in German leagues, namely the "Bundesliga" (men's premier league (West) Germany, 1963/1964 - 2004/2005, ₹ 12800 matches), the "Oberliga" (men's premier league East Germany, $1949 / 1950$ - 1990/1991, $\approx 7700$ matches), and the "Frauen-Bundesliga" (women's premier league Germany, $1997 / 1998$ - 2004/2005, $\approx 1050$ matches) [18]. We determined histograms estimating the probability density functions (PDFs) $P^{\mathrm{h}}\left(n_{h}\right)$ and $P^{\mathrm{a}}\left(n_{a}\right)$ of the final scores of the home and away teams, respectively [19]. Error estimates on the histogram bins were computed with the bootstrap resampling method. This allows the judgment of the quality of the various fits collected in Table 1 by monitoring their goodness or $\chi^{2}$ per degree-of-freedom, $\tilde{\chi}^{2}=\chi^{2}$ d.o.f., naturally taking into account the different numbers of free parameters in the fits considered.

We first considered fits of the PDFs of the phenomenological descriptions (11), (2), and (3). Not to our surprise, 
Table 1: Fits and their $\chi^{2}$ per degree-of-freedom, $\tilde{\chi}^{2}=\chi^{2} /$ d.o.f., of the phenomenological distributions (1), (2), and (3) as well as fits of our microscopic feedback models "A" and "B" to the data for the East German "Oberliga", the (West) German men's premier league "Bundesliga", the German women's premier league "Frauen-Bundesliga" and the qualification stages of all past "FIFA World Cups".

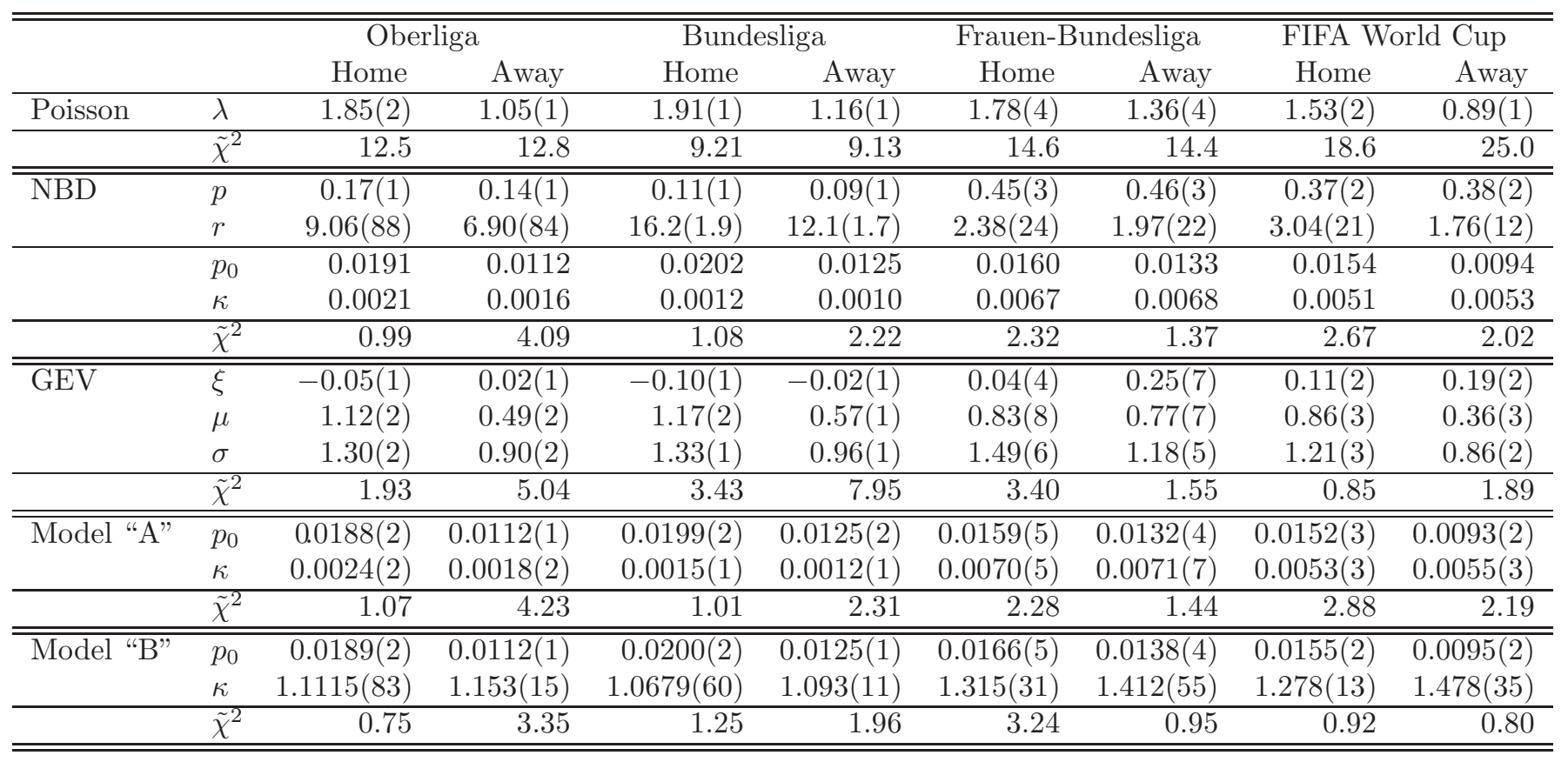

and in accordance with previous findings [6,7], the simple Poissonian ansatz (11) is not found to be an adequate description for any of the data sets. Deviations occur here mainly in the tails with large numbers of goals which in general are found to be fatter than can be accommodated by a Poissonian model. On the contrary, the NBD form (2) models all of the above data well as is illustrated in Fig. 1. Considering the fits of the GEV distributions (3), we find that extreme value statistics are in general also a reasonably good description of the data. The shape parameter $\xi$ is always found to be small in modulus and negative in the majority of the cases, indicating a distribution of the Weibull type (which is in agreement with the findings of Ref. [7] for different leagues). Fixing $\xi=0$ yields overall clearly larger $\chi^{2}$ values. Comparing "Oberliga" and "Bundesliga", we consistently find larger values of the parameter $\xi$ for the former, indicative of the comparatively fatter tails of these data, see Table 1 and Fig. 1. Comparing to the results for the NBD, we do not find any cases where the GEV distributions would provide the best fit to the data, so clearly the leagues considered here are not of the type for which Greenhough et al. [7] found better matches with the GEV statistics than for the NBD. Similar conclusions hold true for the comparison of "Bundesliga" and "Frauen-Bundesliga", with the latter taking on the role of the "Oberliga".

Representing the continuum limit of our model "A", the good performance of the NBD fits observed so far im- plies that the feedback models proposed here can indeed capture the main characteristics of the game. To test this conjecture directly we performed fits of the exact distributions resulting from the recurrence relation (4), employing the simplex method to minimize the total $\chi^{2}$ deviation for the home and away scores. Comparing the results of model "A" to the fits of the limiting NBD, we observe in Table 1 almost identical fit qualities for the final scores. However, for sums and differences of scores we find a considerably better description by using our model "A", indicating deviations from the continuum limit there [17]. The overall best modeling of the league data is achieved with fits of model "B" which feature on average an even higher quality than those of model "A", cf. Table 1 We also performed fits to the more elaborate model "C", but found the results rather similar to those of the simpler model "B" and hence do not discuss them here.

Comparing the leagues, we see in Table 1 that the parameters $\kappa$ for the "Oberliga" are significantly larger than for the "Bundesliga", whereas the parameters $p_{0}$ are slightly smaller for the "Oberliga". That is to say, scoring a goal in a match of the East German "Oberliga" was a more encouraging event than in the (West) German "Bundesliga". Alternatively, this observation might be interpreted as a stronger tendency of the perhaps more professionalized teams of the (West) German premier league to switch to a strongly defensive mode of play in case of a lead. Consequently, the tails of the distributions 

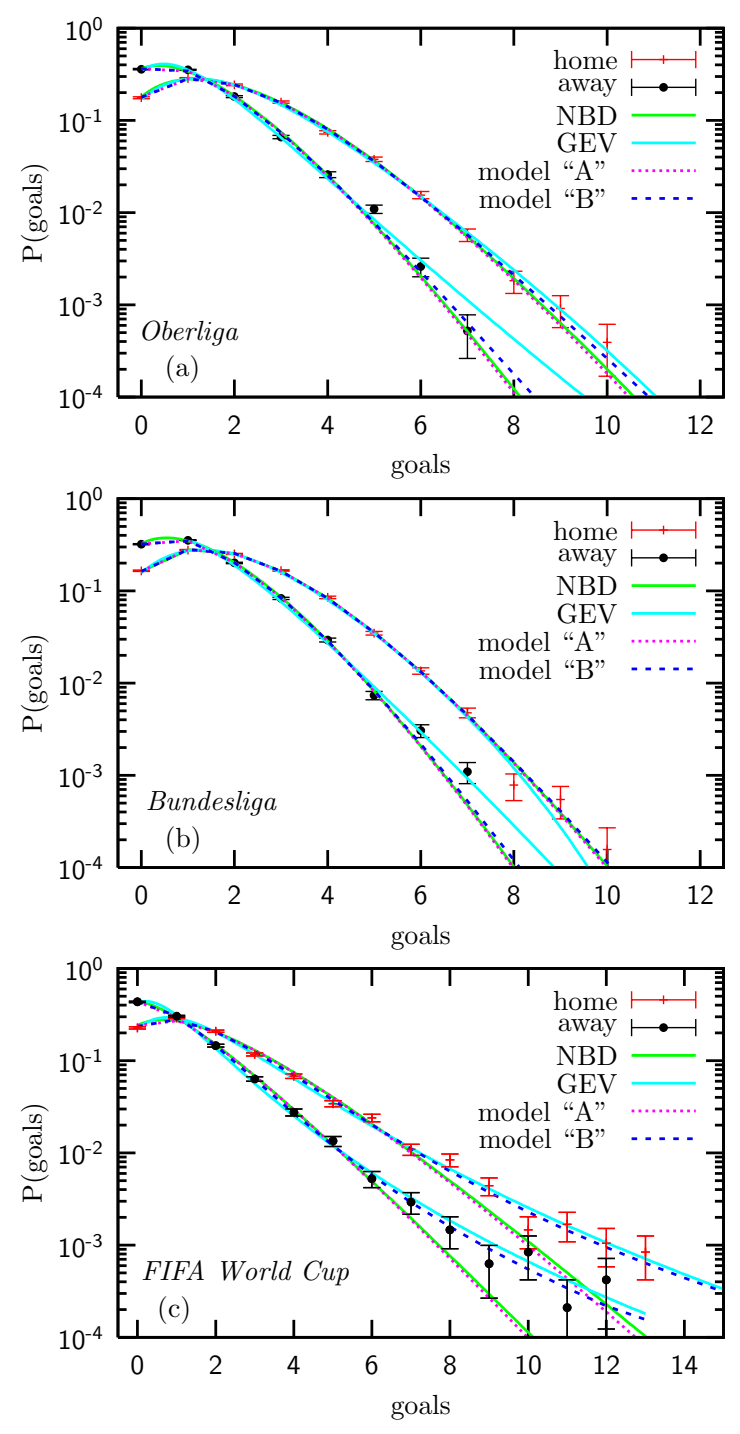

Fig. 1: Histograms of final scores of home and away teams and corresponding fits. (a) East German "Oberliga". (b) (West) German "Bundesliga". (c) The qualification stage of the "FIFA World Cup" series.

are slightly fatter for the "Oberliga" than for the "Bundesliga". Recalling that the NBD form (2) is the continuum limit of the feedback model "A", these differences should translate into larger values of $r$ and smaller values of $p$ for the "Bundesliga" results, which is what we indeed observe. Conversely, computing from the NBD parameters $r$ and $p$ the feedback parameters $p_{0}$ and $\kappa$ also given in Table 1. we obtain good agreement with the directly fitted values. Comparing the results for the "Frauen-Bundesliga" to those for the "Bundesliga", even more pronounced tails are found for the former, resulting in very significantly larger values of the self-affirmation parameter $\kappa$.

Finally, we also considered the score data of the qualification stage of the "FIFA World Cup" series from 1930 to 2002 ( $\approx 3400$ matches) $[20,21]$. Compared to the domes- tic league data discussed above, the results of the World Cup show distinctly heavier tails, cf. Fig. 1. Consequently we obtain good fits for the heavy-tailed distributions, and, in particular, in this case the GEV distribution provides a better fit than the NBD, similar to what was found by Greenhough et al. [7], cf. Table 1] The fits of model "A" are again rather similar to the NBD. The multiplicative feedback model "B", on the other hand, also handles this case extremely well and, for the away team, considerably better than the GEV distribution (3). The difference to the league data can be attributed to the possibly very large differences in skill between the opposing teams occurring since all countries are allowed to participate in the qualification round. The parameters in Table 1 reveal a remarkable similarity with the parameters of the "FrauenBundesliga", where a similar explanation appears quite plausible since the very good players are concentrated in just two or three teams.

We have shown that football score data can be understood from a certain class of modified binomial models with a built-in effect of self-affirmation of the teams upon scoring a goal. The NBD fitting many of the data sets can in fact be understood as a limiting distribution of our model "A" with an additive update rule of the scoring probability. It does not provide very good fits in cases with heavier tails, such as the qualification round of the "FIFA World Cup" series. The overall best variant is our model "B", where a multiplicative update rule ensures that each goal motivates the team even more than the previous one. Basically by "interpolating" between the GEV form and NBD, it fits both these world-cup data as well as the data from the German domestic leagues extremely well, thus reconciling the heterogeneous phenomenological findings with a plausible and simple microscopic model. In general, we find less professionalized leagues or cups to feature stronger scoring feedback, resulting in goal distributions with heavier tails. It is obvious that the presented models with a single parameter of self-affirmation are a bold simplification. It is all the more surprising then, how rather well they model the considered score data, yielding a new example of how sums of correlated variables lead to non-Gaussian distributions with fat tails. For a closer understanding of the self-affirmation effect, an analysis of time-resolved scoring data would be highly desirable. Some data of this type has been analyzed in Ref. [22], showing a clear increase of scoring frequency as the match progresses, thus supporting the presence of feedback as discussed here.

$$
* * *
$$

The authors are grateful to O. Penrose and S. Zachary for discussions. This work was partially supported by the DFG under grant Nos. JA483/22-1 \& 23-1 and the EC RTN-Network 'ENRAGE': Random Geometry and Random Matrices: From Quantum Gravity to Econophysics under grant No. MRTN-CT-2004-005616. M.W. acknowl- 
edges support by the EC MC-EIF programme under contract No. MEIF-CT-2004-501422.

\section{REFERENCES}

[1] D. Stauffer, Physica A336 (2004) 1.

[2] B. K. Chakrabarti, A. Chakraborti, and A. Chatterjee, (ed.) Econophysics and Sociophysics: Trends and Perspectives (Wiley-VCH, Berlin, 2006).

[3] S. R. Clarke and J. M. Norman, The Statistician 45 (1995) 509; L. C. Malacarne and R. S. Mendes, Physica A286 (2000) 391; S. Dobson and J. Goddard, Eur. J. Oper. Res. 148 (2003) 247; J. Goddard and I. Asimakopoulos, J. Forecast. 23 (2004) 51; R. N. Onody and P. A. de Castro, Phys. Rev. E70 (2004) 037103; N. P. Linthorne and D. J. Evertt, Sports Biomechanics 5 (2006) 5; J. Sprem, Simulation der Fußball-Bundesliga, Master's thesis, Universität zu Köln (2006).

[4] M. J. Moroney, Facts from Figures, 3rd edition (Penguin, London, 1956).

[5] J. Wessons, The Science of Soccer (Institute of Physics Publishing, Bristol, 2002).

[6] C. Reep, R. Pollard, and B. Benjamin, J. Roy. Stat. Soc. A134 (1971) 623.

[7] J. Greenhough, P. C. Birch, S. C. Chapman, and G. Rowlands, Physica A316 (2002) 615.

[8] S. Kotz and S. Nadarajah, Extreme Value Distributions: Theory and Applications (World Scientific, Singapore, 2000).

[9] S. T. Bramwell, P. C. W. Holdsworth, and J.-F. Pinton, Nature 396 (1998) 552; S. T. Bramwell, K. Christensen, J.-Y. Fortin, P. C. W. Holdsworth, H. J. Jensen, S. Lise, J. M. López, M. Nicodemi, J.-F. Pinton, and M. Sellitto, Phys. Rev. Lett. 84 (2000) 3744.

[10] J.-P. Bouchaud and M. Mézard, J. Phys. A30 (1997) 7997.

[11] B. A. Berg and W. Janke, Phys. Rev. Lett. 80 (1998) 4771; B. A. Berg, A. Billoire, and W. Janke, Phys. Rev. E65 (2002) 045102(R).

[12] E. Bittner and W. Janke, Europhys. Lett. 74 (2006) 195.

[13] A. Noullez and J.-F. Pinton, Eur. Phys. J. B28 (2002) 231.

[14] P. A. Varotsos, N. V. Sarlis, H. K. Tanaka, and E. S. Skordas, Phys. Rev. E72 (2005) 041103.

[15] E. Bertin, Phys. Rev. Lett. 95 (2005) 170601; E. Bertin and M. Clusel, J. Phys. A39 (2006) 7607.

[16] W. Feller, An Introduction to Probability Theory and its Applications, vol. 1, 3rd edition (Wiley, New York, 1968).

[17] E. Bittner, A. Nußbaumer, W. Janke, and M. Weigel, Preprint physics/0606016.

[18] www.fussballdaten.de; www.fussballportal.de; www.nordostfussball.de; www.sportergebnise.de.

[19] To ensure reliable error estimates, in the fits presented below we ignored histogram bins consisting of single or isolated entries, i.e., outliers.

[20] www.rdasilva.demon.co.uk/football.html.

[21] We disregarded all games played in tournaments on neutral grounds. The final knockout stage follows different rules [17].

[22] M. J. Dixon and M. E. Robinson, The Statistician 47 (1998) 523. 